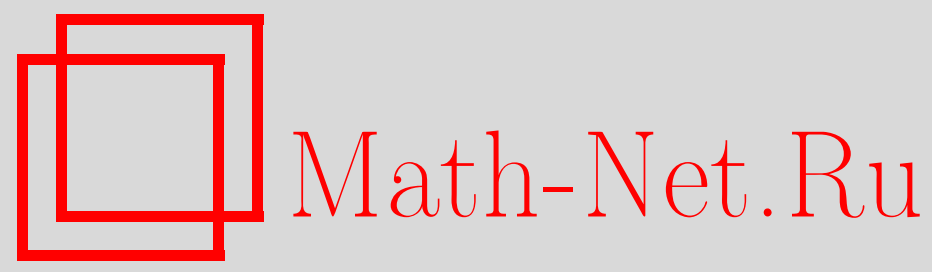

В. С. Самовол, Нормальная форма автономной системы с одним нулевым корнем, Матем. заметки, 2004, том 75, выпуск $5,711-720$

DOI: https://doi.org/10.4213/mzm67

Использование Общероссийского математического портала Math-Net.Ru подразумевает, что вы прочитали и согласны с пользовательским соглашением http://www.mathnet.ru/rus/agreement

Параметры загрузки:

IP : 18.209 .158 .208

26 апреля 2023 г., $12: 47: 55$ 


\title{
НОРМАЛЬНАЯ ФОРМА АВТОНОМНОЙ СИСТЕМЫ С ОДНИМ НУЛЕВЫМ КОРНЕМ
}

\author{
В. С. Самовол
}

\begin{abstract}
В статье рассматриваются задачи гладкой нормализации систем обыкновенных дифференциальных уравнений, линейная часть которых имеет одно нулевое собственное число, в то время как другие собственные числа лежат вне мнимой оси.

Библиография: 7 названий.
\end{abstract}

Введение. В работе рассматривается задача локальной конечно-гладкой приводимости вещественной автономной системы обыкновенных дифференциальных уравнений к нормальной форме в окрестности особой точки. Речь пойдет о системах, матрища линейной части которых имеет одно нулевое собственное число, в то время как другие собственные числа лежат вне мнимой оси. Нормальная форма систем обыкновенных дифференциальных уравнений достаточно хорошо изучена. В частности, подробно исследована задача аналитической приводимости к нормальной форме (см. работы А. Д. Брюно; например, [1]). В данной работе нас будет интересовать задача как о собственно нормальной форме, так и о конечно-гладкой приводимости к ней систем гладких дифференциальных уравнений. Такие задачи также хорошо изучены, однако в большинстве работ исследуются системы с невырожденной особой точкой (или инвариантным многообразием) в то время как даже слабо вырожденные системы весьма мало изучены. По поводу частично вырожденных систем см. [2]. Одна из первых попыток исследования указанных систем представлена в [3].

Рассмотрим вещественную автономную систему

$$
\dot{\xi}=\frac{d \xi}{d t}=Q(\xi)
$$

где $\xi, Q(\xi) \in \mathbb{R}^{n+1}, n>0, Q(\xi)$ - функция класса $C^{\infty}$ в некоторой окрестности начала координат, $Q(0)=0$, матрища $\widetilde{A}=Q^{\prime}(0)$ имеет $n$ собственных чисел, лежаших вне мнимой оси и одно нулевое собственное число. Цель данной работы состоит в определении вида нормальной формы такой системы уравнений и в выяснении условий существования конечно-гладкого невырожденного преобразования, приводящего систему (1) к нормальной форме в некоторой окрестности начала координат. Эту задачу мы будем называть задачей локальной конечно-гладкой нормализачии системы (1).

Работа выполнена при финансовой поддержке Российского фонда фундаментальных исследований, грант № 03-01-00426. 
Пусть $\lambda_{1}, \ldots, \lambda_{n}$ - ненулевые собственные числа матрицы $\widetilde{A}, \lambda_{n+1}=0$. С помошью стандартного линейного преобразования приведем систему (1) к следующему виду, где матрица $\widetilde{A}$ имеет жорданову форму:

$$
\begin{aligned}
\frac{d x_{i}}{d t} & =\varepsilon_{i} x_{i-1}+\lambda_{i} x_{i}+f_{i}(x, y), \quad i=1, \ldots, n, \\
\frac{d y}{d t} & =g(x, y) .
\end{aligned}
$$

Здесь $x_{i}$ - комплексные координаты, при этом, как обычно в подобных случаях, комплексно сопряженньм собственным числам соответствуют комплексно сопряженные уравнения системы; комплексная сопряженность координат и уравнений при всех преобразованиях будет сохраняться. Таким образом, преобразованиям системы (2) будут соответствовать вещественные преобразования системы (1).

В дальнейшем ограничимся рассмотрением системы $(2)$, где $g(0, y)=b y^{m+1}+$ $o\left(|y|^{m+1}\right), b \neq 0, m \geqslant 1$ - некоторое целое число.

Основные результаты работы заключаются в следующих теоремах.

Теорема 1. Для любого целого числа $k>0$ существует невырожденное преобразование класса $C^{k}$, приводящее систему (2) к нормальной форме следующего вида (для простоты мы сохраняем обозначения системы (2)):

$$
\begin{aligned}
\frac{d x_{i}}{d t} & =\varepsilon_{i} x_{i-1}+\lambda_{i} x_{i}+\sum_{j=1}^{n} a_{j}^{i}(y) x_{j}+\sum_{p=2}^{N} a_{p}^{i}(x, y), \quad i=1, \ldots, n \\
\frac{d y}{d t} & =y\left(b y^{m}+c y^{2 m}\right)+\sum_{p=2}^{N} a_{p}^{n+1}(x, y)
\end{aligned}
$$

где $a_{j}^{i}(y)-$ функиии класса $C^{\infty}, a_{j}^{i}(0)=0, a_{j}^{i}(y)=0$, если $\lambda_{j} \neq \lambda_{i}, a_{p}^{i}(x, y)=$ $\sum_{|s|=p} a_{s}^{i}(y) x^{s}, a_{s}^{i}(y)$ - функиии класса $C^{\infty}, s=\left(s_{1}, \ldots, s_{n}\right)$ - наборь неотрицательных иелых чисел, $|s|=\sum_{q=1}^{n} s_{q}-$ вес набора $s, x^{s}=x_{1}^{s_{1}} \cdots x_{n}^{s_{n}}$. При $1 \leqslant i \leqslant n+1 \quad a_{s}^{i}(y)=0$, если $\lambda_{i} \neq \sum_{q=1}^{n} s_{q} \lambda_{q}$. Число $N=N(k)$, причем $\lim _{k \rightarrow \infty} N(k)=\infty$.

Система (3) представляет собой резонансную нормальную форму полиномиального вида по "невырожденньм" координатам, степень которой по этим координатам не превышает числа $N=N(k)$.

Как будет видно из примеров, приведенных в данной статье, интерес представляют такие системы (2), у которых все собственные числа $\lambda_{i}$ различны. Для таких систем верны следующие теоремы.

Теорема 2. Если в системе (2) выполняется условие $\lambda_{i} \neq \lambda_{j}$ при $i \neq j$, то существует формальное преобразование, приводящее систему (2) к формальной нормальной форме следующего вида (для простоты мы сохраняем обозначения системы (3)):

$$
\begin{aligned}
\frac{d x_{i}}{d t} & =\lambda_{i} x_{i}+a_{i}(y) x_{i}+\sum_{p=2}^{\infty} a_{p}^{i}(x, y), \quad i=1, \ldots, n \\
\frac{d y}{d t} & =y\left(b y^{m}+c y^{2 m}\right)+\sum_{p=2}^{\infty} a_{p}^{n+1}(x, y)
\end{aligned}
$$


где $a_{i}(y)$ - многочлены степени т без свободных членов, $a_{p}^{i}(x, y)=\sum_{|s|=p} a_{s}^{i}(y) x^{s}$, $a_{s}^{i}(y)$ - многочлены степени $N_{1}$, число $N_{1}=N_{1}(s)$, причем эта зависимость имеет линейный характер. При $1 \leqslant i \leqslant n+1 \quad a_{s}^{i}(y)=0$, если $\lambda_{i} \neq \sum_{q=1}^{n} s_{q} \lambda_{q}$.

Теорема 3. Если в системе (2) выполняется условие $\lambda_{i} \neq \lambda_{j}$ при $i \neq j$, то для любого челого числа $k>0$ существует невырожденное преобразование класса $C^{k}$, приводящее систему (2) к нормальной форме следующего вида (для простоты мы сохраним обозначения системы (4)):

$$
\begin{aligned}
\frac{d x_{i}}{d t} & =\lambda_{i} x_{i}+a_{i}(y) x_{i}+\sum_{p=2}^{N} a_{p}^{i}(x, y), \quad i=1, \ldots, n, \\
\frac{d y}{d t} & =y\left(b y^{m}+c y^{2 m}\right)+\sum_{p=2}^{N} a_{p}^{n+1}(x, y),
\end{aligned}
$$

где $a_{i}(y)$ - многочлены степени т без свободных членов, $a_{p}^{i}(x, y)=\sum_{|s|=p} a_{s}^{i}(y) x^{s}$, $a_{s}^{i}(y)$-многочлены степени $N_{1}(s)$. При $1 \leqslant i \leqslant n+1 \quad a_{s}^{i}(y)=0$, если $\lambda_{i} \neq \sum_{q=1}^{n} s_{q} \lambda_{q}$. Число $N=N(k)$.

1. Вспомогательные утверждения. На одномерном центральном многообразии, соответствующем нулевому собственному значению, система (2) может быть приведена невырожденным преобразованием класса $C^{\infty}$ к виду (см., например, [4], [5])

$$
\frac{d y}{d t}=y\left(b y^{m}+c y^{2 m}\right) .
$$

Ниже будем считать, что в системе (2) это преобразование уже сделано.

Займемся теперь преобразованием линейной (по невырожденным координатам) части системы (2). Для этого рассмотрим соответствующую систему уравнений:

$$
\begin{aligned}
\frac{d z_{i}}{d t} & =\varepsilon_{i} z_{i-1}+\lambda_{i} z_{i}+\sum_{j=1}^{n} f_{j}^{i}(y) z_{j}, \quad i=1, \ldots, n, \\
\frac{d y}{d t} & =y\left(b y^{m}+c y^{2 m}\right),
\end{aligned}
$$

где $f_{j}^{i}(y)=\partial f_{i}(z, y) / \partial z_{j}$ при $z=0$.

Лемма 1. Для системы (7) существует невырожденное преобразование класca $C^{\infty}$

$$
z=x+H(y) x, \quad H(0)=0,
$$

приводящее ее к следующей нормальной форме:

$$
\begin{aligned}
\frac{d x_{i}}{d t} & =\varepsilon_{i} x_{i-1}+\lambda_{i} x_{i}+\sum_{j=1}^{n} a_{j}^{i}(y) x_{j}, \quad i=1, \ldots, n, \\
\frac{d y}{d t} & =y\left(b y^{m}+c y^{2 m}\right),
\end{aligned}
$$

которая отличается от системы (3) лишь отсутствием нелинейных по переменным $x_{1}, \ldots, x_{n}$ членов. 
ДокАЗАТЕЛьство. Известно (см., например [1]), что существует формальное (не обязательно сходящееся) преобразование вида (8), в результате которого система (7) формально приводится к нормальной форме вида $(9)$, где $a_{j}^{i}(y)$ - формальные ряды. Это означает, что существует (уже не формальное) преобразование класса $C^{\infty}$, ряд Тейлора которого совпадает с вышеуказанным формальным преобразованием, в результате которого система (7) приобретет следующий вид:

$$
\begin{aligned}
\frac{d u_{i}}{d t} & =\varepsilon_{i} u_{i-1}+\lambda_{i} u_{i}+\sum_{j=1}^{n} a_{j}^{i}(y) u_{j}+\sum_{j=1}^{n} c_{j}^{i}(y) u_{j}, \quad i=1, \ldots, n \\
\frac{d y}{d t} & =y\left(b y^{m}+c y^{2 m}\right)
\end{aligned}
$$

где $a_{j}^{i}(y)$ - функции класса $C^{\infty}, a_{j}^{i}(y)=0$, если $\lambda_{i} \neq \lambda_{j}$, a $c_{j}^{i}(y)$ - плоские функции.

Будем теперь искать преобразование, приводящее систему (10) к ее нормальной форме (9) в виде

$$
u=x+H(y) x
$$

Подставляя (11) в (10) и учитьвая систему (9), получаем, что матрица $H(y)$ должна удовлетворять уравнению

$$
\begin{aligned}
\dot{H} & =\left(A+A_{1}(y)\right) H-H\left(A+A_{1}(y)\right)+C(y) H+C(y), \\
\dot{y} & =y\left(b y^{m}+c y^{2 m}\right),
\end{aligned}
$$

где $A$ - постоянная матрица правой части системы $(10)$; элементами матрищы $A_{1}(y)$ являются функции $a_{j}^{i}(y)$ системы $(10)$, а элементы матрицы $C(y)$ - это функции $c_{j}^{i}(y)$ той же системы.

Рассматривая элементы искомой матрицы $H(y)$ в качестве компонент вектора $h(y)$, получаем, что этот вектор должен удовлетворять системе уравнений вида

$$
\begin{aligned}
& \dot{h}=\widetilde{A}(y) h+\alpha(y), \\
& \dot{y}=y\left(b y^{m}+c y^{2 m}\right),
\end{aligned}
$$

где $\widetilde{A}(y)$ - матрица класса $C^{\infty}$, а $\alpha(y)$ - плоская вектор-функция. Из работы [6] следует, что у системы (13) имеется плоское решение $h(y)$ класса $C^{\infty}$, что завершает доказательство леммы.

Рассмотрим случай системы (7), которая удовлетворяет следующему дополнительному условию $\lambda_{i} \neq \lambda_{j}$ при $i \neq j$. В этом случае имеет место следующее утверждение.

Лемма 2. Если в системе (7) все собственные числа $\lambda_{i}, 1 \leqslant i \leqslant n$, различны, то в нормальной форме (9) системы (7) функиии $a_{j}^{i}(y)$ являются полиномами степени не выше $m$. 
ДоКАЗАТЕЛЬСТво. Прежде всего будем считать, что система (7) уже приведена к нормальной форме $(9)$, где функции $a_{j}^{i}(y)$ принадлежат классу $C^{\infty}$. Отметим, что вследствие нашего предположения первые $n$ уравнений системы (9) имеют вид

$$
\frac{d x_{i}}{d t}=\lambda_{i} x_{i}+a_{i}(y) x_{i}, \quad i=1, \ldots, n .
$$

Представим функции $a_{i}(y)$ в виде $a_{i}(y)=\sum_{p=1}^{m} c_{i p} y^{p}+y^{m+1} a_{i 1}(y)$, где $a_{i 1}(y)$ - функции класса $C^{\infty}$. Сделаем преобразование $x_{i}=u_{i}+h_{i}(y) u_{i}$ в системе $(9)$. Получим

$$
\frac{d u_{i}}{d t}=\lambda_{i} u_{i}+\left(\sum_{p=1}^{m} c_{i p} y^{p}\right) u_{i}+y^{m+1} a_{i 1}(y) u_{i}-\frac{h_{i}(y) u_{i}}{1+h_{i}(y)}, \quad i=1, \ldots, n .
$$

В качестве функций $h_{i}(y)$ необходимо взять функции, удовлетворяюшие уравнению

$$
\frac{d h_{i}}{d y}=\frac{\left(1+h_{i}\right) a_{i 1}(y)}{b+c y^{m}} .
$$

Очевидно, данное уравнение имеет решение класса $C^{\infty}$. Лемма 2 доказана.

Отметим, что лемма 2 следует из теоремы 3 п. 2.3. главы 3 книги [1].

Условие $\lambda_{i} \neq \lambda_{j}$ при $i \neq j$ является существенным. Если оно не выполняется, то нельзя утверждать приводимость к указанной нормальной форме с помошюю преобразования класса $C^{\infty}$. Это показывает пример следующей системы.

ПРИМЕР 1. Рассмотрим систему

$$
\begin{aligned}
\dot{x}_{1} & =x_{1}+y^{2} x_{2}, \\
\dot{x}_{2} & =x_{2}-y x_{2}, \\
\dot{y} & =y^{2} .
\end{aligned}
$$

Прямая проверка показьвает, что эта система не может быть приведена преобразованием класса $C^{\infty}$ к нормальной форме, не содержащей в первом уравнении нелинейньх членов по переменной $y$. Действительно, допустим, что существует невырожденноепреобразование, приводящее данную систему к искомой форме. Без ограничения общности можно ограничиться рассмотрением лишш преобразований, близких к тождественным (это показьвается так же, как и в [7, с. 69]). Предположим теперь, что в результате следующей замены переменных:

$$
x=u+y B_{1} u+y^{2} B_{2} u+H(y) u, \quad H(y)=o\left(y^{2}\right),
$$

система (14) приобретет требуемую форму

$$
\dot{u}=u+y D u,
$$

где $D$ - некоторая постоянная матрица. Подставляя (15) в систему (14) и учитывая (16), приравняем вначале члены линейные по переменной $y$. В результате получаем равенство $D=C_{1}$, где матрица $C_{1}=\left(\begin{array}{cc}0 & 0 \\ 0 & -1\end{array}\right)$. Приравнивая теперь члены второй степени по переменной $y$, получаем, что матрица $B_{1}$ должна удовлетворять равенству

$$
B_{1} \cdot C_{1}+B_{1}=C_{2} \cdot B_{1}+C_{3},
$$

где $C_{2}=\left(\begin{array}{cc}0 & 0 \\ 0 & -1\end{array}\right), C_{3}=\left(\begin{array}{ll}0 & 1 \\ 0 & 0\end{array}\right)$.

Приравнивая элементы, находящиеся в первой строке и втором столбце матриц в равенстве (17) получаем, что $0=1$. Данное противоречие доказывает невозможность преобразования (15). 
2. Конечно-гладкая нормализация. В этом разделе мы приведем доказательство теоремы 1 .

ДоКАЗАТЕЛЬСТВо ТЕОРЕМЫ 1. Предположим, что система (2) невырожденньм преобразованием класса $C^{\infty}$ может быть приведена к следующему виду (обозначения те же, что и в формуле (3)):

$$
\begin{aligned}
\frac{d x_{i}}{d t} & =\varepsilon_{i} x_{i-1}+\lambda_{i} x_{i}+\sum_{p=1}^{l+1} a_{p}^{i}(x, y)+\alpha_{i}(x, y), \quad i=1, \ldots, n \\
\frac{d y}{d t} & =y\left(b y^{m}+c y^{2 m}\right)+\sum_{p=1}^{l+1} a_{p}^{n+1}(x, y)+\alpha_{n+1}(x, y)
\end{aligned}
$$

где $l \geqslant 1$ - целое число; $\alpha_{i}(x, y)=o\left(\|x\|^{l+1}\right) ;$ при $|s| \leqslant l$ вьполнены условия

$$
a_{s}^{i}(y)=0, \quad \text { если } \quad \sum_{p=1}^{n} s_{p} \lambda_{p} \neq \lambda_{i}, \quad 1 \leqslant i \leqslant n+1 .
$$

Напомним, что $a_{p}^{i}(x, y)=\sum_{|s|=p} a_{s}^{i}(y) x^{s}$.

При $l=1$ данное предположение следует из леммы 1 . Пусть оно верно при некотором $l \geqslant 1$. Покажем, что систему (18) можно привести к такому же виду, где условия (19) будут выполнены уже при $|s| \leqslant l+1$.

Для этого вначале для каждого нерезонансного члена вида $c_{s}^{i}(y) x^{s},|s|=l+1$, построим замену переменных, в результате которой данньй нерезонансньй член приобретет вид $\alpha_{s}^{i}(y) x^{s}$, где $\alpha_{s}^{i}(y)$ уже будет плоской функцией. Затем применим преобразование, использованное в доказательстве леммы 1 , в результате чего указанные резонансные члены исчезнут и система приобретет вид (18), где условия (19) будут выполнены уже при $|s| \leqslant l+1$.

Пусть теперь в системе (18) условия (19) выполнены для $|s| \leqslant N$, где $N$ достаточно большое число. Более точно, пусть выполнено условие $N=N(k, \Lambda)$, где $\Lambda=\left(\lambda_{1}, \ldots\right.$, $\left.\lambda_{n}\right)$ - спектр матрицы $A, k \geqslant 1$ - целое число, а число $N(k, \Lambda)$ определено в теореме 1 работы [2]. Тогда для системы (18), где условия (19) выполнены при $|s| \leqslant N(k, \Lambda)$, в соответствии с указанной теоремой существует близкое к тождественному преобразование класса $C^{k}$, в результате применения которого система (18) примет требуемьй вид (3). Теорема 1 доказана.

3. Формальная нормализация систем с различными собственными числами. Рассмотрим такую систему (2), у которой все собственные числа $\lambda_{i}$ различны. В данном разделе мы ограничимся формальньми преобразованиями формальных систем вида (2), имея в виду, что правые части этих систем представляют собой формальные степенные ряды. Покажем теперь, что для таких систем справедлива теорема 2, согласно которой система (2) формально приводима к нормальной форме вида (4).

ДоКАЗАТЕЛЬСТВО ТЕОРЕМЫ 2. Используя стандартные рассуждения, приведенные в доказательстве теоремы 1 , приходим к выводу, что система (2) формально при- 
водима к нормальной форме следующего вида:

$$
\begin{aligned}
\frac{d x_{i}}{d t} & =\lambda_{i} x_{i}+a_{i}(y) x_{i}+\sum_{p=2}^{\infty} a_{p}^{i}(x, y), \quad i=1, \ldots, n \\
\frac{d y}{d t} & =y\left(b y^{m}+c y^{2 m}\right)+\sum_{p=2}^{\infty} a_{p}^{n+1}(x, y)
\end{aligned}
$$

где $a_{p}^{i}(x, y)=\sum_{|s|=p} a_{s}^{i}(y) x^{s}, a_{i}(y), a_{s}^{i}(y)$ - формальные степенные ряды, $1 \leqslant i \leqslant n+1$. Согласно лемме 2 система (2) может быть формально приведена к виду, где функции $a_{i}(y)$ являются полиномами степени не вьше $m$. Будем считать, что это условие в системе (20) вьполняется. Покажем теперь существование формального преобразования, после которого функции $a_{s}^{i}(y)$ также окажутся полиномами. Зафиксируем некоторьй резонансньй набор $s,|s|>1$, для которого $a_{s}^{i}(y) \neq 0$. Представим функцию $a_{s}^{i}(y)$ в следующем виде:

$$
a_{s}^{i}(y)=\sum_{q=0}^{N} a_{q} y^{q}+\sum_{q=N+l}^{\infty} a_{q} y^{q}
$$

где число $N=N_{1}$ мы определим позже, а число $l \geqslant 1$. Пока ограничимся тем, что будем считать, что $N_{1} \geqslant 2 m$.

Рассмотрим два случая. В первом случае будем предполагать, что в последнем уравнении исходной системы $(2)$ коэффициент $c \neq 0$. В этом случае сделаем замену переменных

$$
\begin{aligned}
& x_{i}=u_{i}+d \cdot y^{N+l-2 m} u^{s}, \\
& x_{j}=u_{j}, \quad j \neq i .
\end{aligned}
$$

Подставляя (22) в (20), получаем, что в преобразованном уравнении коэффициент при $y^{N+1}$ в формальном ряде, являющемся коэффициентом при мономе $u^{s}$, будет равен $a_{N+l}-(N+l-2 m) \cdot c \cdot d$. Полагая $d=a_{N+l} /((N+l-2 m) \cdot c)$, мы приведем систему к виду, где функция $a_{s}^{i}(y)$ приобретет вид $a_{s}^{i}(y)=\sum_{p=0}^{N} \tilde{a}_{p} y^{p}+\sum_{p=N+l-1}^{\infty} \tilde{a}_{p} y^{p}$, причем коэффициент $\tilde{a}_{N+l}=0$. Повторяя соответствующее преобразование конечное число раз, приводим в итоге систему к виду, где функция $a_{s}^{i}(y)$ будет представлять собой сумму многочлена степени $N$ и формального ряда, наименьшая степень которого равна $N+l+1$. Таким образом, можно считать, что в нашей системе функция $a_{s}^{i}(y)$ имеет вид $a_{s}^{i}(y)=\sum_{q=0}^{N} a_{q} y^{q}+\sum_{q=N+l}^{\infty} a_{q} y^{q}$, где уже $l \geqslant 2$. Проводя индукцию по $l$, получаем существование формального преобразования, приводящего систему к виду, где функция $a_{s}^{i}(y)=\sum_{q=0}^{N} a_{q} y^{q}$. Подобное преобразование можно провести для любого резонансного набора $s$. В итоге мы получаем формальную приводимость системы (2) к виду (5), где функции $a_{s}^{i}(y)$ являются многочленами степени $N_{1}=2 m$.

Рассмотрим теперь случай, когда в последнем уравнении исходной системы (2) коэффициент $c=0$.

По-прежнему считаем, что функция $a_{s}^{i}(y)$ имеет вид $(21)$, где число $N=N_{1}(s)$ мы определим позже. В этом случае сделаем замену переменньх

$$
\begin{aligned}
& x_{i}=u_{i}+d \cdot y^{N+l-m} u^{s}, \\
& x_{j}=u_{j}, \quad j \neq i .
\end{aligned}
$$


Подставляя (23) в (20), получаем, что в преобразованном уравнении коэффициент при $y^{N+1}$ в формальном ряде, являющемся коэффициентом при мономе $u^{s}$, будет равен

$$
a_{N+l}-\left((N+l-m) \cdot b+\left\langle s, a_{m}\right\rangle-a_{i m}\right) \cdot d,
$$

где $\left\langle s, a_{m}\right\rangle$ - это скалярное произведение вектора $s$ и вектора $a_{m}=\left(a_{1 m}, \ldots, a_{n m}\right)$, координаты которого $a_{i m}$ являются коэффициентами при $y^{m}$ в функциях $a_{i}(y), 1 \leqslant i \leqslant n$. Полагая число

$$
d=\frac{a_{N+l}}{(N+l-m) \cdot b+\left\langle s, a_{m}\right\rangle-a_{i m}}
$$

мы приведем систему к виду, где функция $a_{s}^{i}(y)$ приобретет вид $a_{s}^{i}(y)=\sum_{p=0}^{N} \tilde{a}_{p} y^{p}+$ $\sum_{p=N+l-1}^{\infty} \tilde{a}_{p} y^{p}$, причем коэффициент $\tilde{a}_{N+l}=0$. Указанное число $d$ существует, если в определяющей его дроби знаменатель не равен нулю. Если число $N$ выбрать таким, чтобы оно удовлетворяло неравенству

$$
N>\frac{\left|\left\langle s, a_{m}\right\rangle\right|+\max _{1 \leqslant i \leqslant n}\left|a_{i m}\right|}{|b|}+m-1,
$$

то требуемое условие будет вьполнено. Повторяя теперь рассуждения, приведенные выше для случая $c \neq 0$, получаем формальную приводимость системы (2) к виду (5), где функции $a_{s}^{i}(y)$ являются многочленами степени $N_{1}=N_{1}(s)$, где $N_{1}(s)$ представляет собой целую часть числа

$$
\frac{\left|\left\langle s, a_{m}\right\rangle\right|+\max _{1 \leqslant i \leqslant n}\left|a_{i m}\right|}{|b|}+m .
$$

Данная оценка может быть уточнена. Ясно, что число $N_{1}(s)$ должно быть выбрано таким, чтобы числа $\left(N_{1}(s)+1-m\right) \cdot b+\left\langle s, a_{m}\right\rangle-a_{i m} \neq 0$. Теорема 2 доказана.

Следующий пример показьвает существенность оценки чисел $N_{1}(s)$, приведенных в доказательстве теоремы 2 .

ПРИмеР 2. Рассмотрим систему

$$
\begin{aligned}
\frac{d x_{1}}{d t} & =x_{1}+x_{1} y+x_{2}^{p} x_{3}^{q}\left(y^{r}+o\left(y^{r}\right)\right), \\
\frac{d x_{2}}{d t} & =-2 x_{2}-x_{2} y, \\
\frac{d x_{3}}{d t} & =3 x_{3}-x_{3} y, \\
\frac{d y}{d t} & =y^{2} .
\end{aligned}
$$

Здесь $o\left(y^{r}\right)$ представляет собой формальный ряд, слагаемые которого имеют степень выше $r$.

Непосредственная проверка показывает, что эта система не может быть формально приведена к нормальной форме, не содержащей нелинейных по переменной $y$ членов в первом уравнении, степень которых ниже, чем $p+q+2$. Таким образом, если в первом уравнении число $r=p+q+2$, то степень соответствующего многочлена не может 
быть ниже, чем данное число $r$, что показьвает точность полученной в доказательстве теоремы 2 оценки чисел $N_{1}(s)$.

В теореме 2 мы исходили из того, что все собственные числа $\lambda_{i}$ различны. Данное условие обеспечивало формальную приводимость линейной части системы к полиномиальной нормальной форме (лемма 2). Если указанное условие не выполнено, то приведение к такой нормальной форме не всегда возможно. Однако если предположить, что нормальная форма линейной системы (7) имеет вид (9), где функции $a_{j}^{i}(y)$ являются многочленами степени $m$, то теорема 2 остается верной и без вьполнения условия различности чисел $\lambda_{i}$. Это означает, что формальная нормальная форма системы $(2)$ имеет в такой ситуации полиномиальный вид, однако оценка чисел $N_{1}(s)$ будет в этом случае другой.

\section{4. Конечно-гладкая нормализация систем с различными собственными} числами. Данный раздел содержит доказательство теоремы 3.

ДокАЗАТЕЛЬСТво ТЕОРЕМЫ 3. Согласно теореме 2 система (2), у которой все числа $\lambda_{i}$ различны, формально приводима к нормальной форме вида (20). Но тогда с помощью преобразования класса $C^{\infty}$, ряд Тейлора которого совпадает с формальным преобразованием системы (2), мы можем привести указанную систему к виду

$$
\begin{aligned}
\frac{d x_{i}}{d t} & =\lambda_{i} x_{i}+a_{i}(y) x_{i}+a_{i 1}(y) x_{i}+a_{i}^{1}(x, y)+a_{i}^{2}(x, y), \quad i=1, \ldots, n, \\
\frac{d y}{d t} & =y\left(b y^{m}+c y^{2 m}\right)+a_{n+1}^{1}(x, y)+a_{n+1}^{2}(x, y)
\end{aligned}
$$

где функции $a_{i}(y)$ являются многочленами степени $m$, ряды Тейлора функций $a_{i}^{1}(x, y)$ совпадают с формальными рядами $\sum_{|s|=p}^{\infty} a_{p}^{i}(x, y)$, где $a_{p}^{i}(x, y)=\left(a_{s}^{i}(y)+\alpha_{s}^{i}(y)\right) x^{s}$, $a_{s}^{i}(y)$ - многочлены степени $N_{1}(s), a_{i 1}(y), \alpha_{s}^{i}(y)$ - плоские функции, $a_{i}^{2}(x, y)$ - функции, плоские по переменным $x_{1}, \ldots, x_{n}, 1 \leqslant i \leqslant n+1$.

Пусть теперь $k \geqslant 1$ - целое число, а число $N=N(k)=N(k, \Lambda)$, где $\Lambda=\left(\lambda_{i}, \ldots, \lambda_{n}\right)-$ спектр матрицы $A$ и $N(k, \Lambda)$ определено в теореме 1 работы [2]. Тогда для системы $(24)$ в соответствие с указанной теоремой существует близкое к тождественному преобразование класса $C^{k}$, в результате применения которого система (24) приобретет вид

$$
\begin{aligned}
\frac{d x_{i}}{d t} & =\lambda_{i} x_{i}+a_{i}(y) x_{i}+a_{i 1}(y) x_{i}+\tilde{a}_{i}^{1}(x, y), \quad i=1, \ldots, n \\
\frac{d y}{d t} & =y\left(b y^{m}+c y^{2 m}\right)+\tilde{a}_{n+1}^{1}(x, y)
\end{aligned}
$$

где $\tilde{a}_{i}^{1}(x, y)=\sum_{|s|=2}^{N(k)}\left(a_{s}^{i}(y)+\alpha_{s}^{i}(y)\right) x^{s}, 1 \leqslant i \leqslant n+1$.

$\mathrm{C}$ помощью преобразования класса $C^{\infty}$, предложенного в работе [6] и использованного при доказательстве леммы 1 , избавляемся от конечного числа плоских слагаемых в уравнениях системы (25). В результате получаем существование замены переменных класса $C^{\infty}$, после которой система (25) приобретет требуемый вид (5). Теорема 3 доказана.

Автор выражает искреннюю благодарность А.Д. Брюно за полезные обсуждения вопросов, связанных с проблематикой данной работы, в частности, за формулировку теоремы 2 . 


\section{СПИСОК ЦИТИРОВАННОЙ ЛИТЕРАТУРЫ}

[1] Брюно А. Д. Локальный метод нелинейного анализа дифференциальных уравнений. М.: Наука, 1979.

[2] Самовол В. С. Эквивалентность систем дифференциальных уравнений в окрестности особой точки // Тр. ММО. 1982. Т. 44. С. 213-234.

[3] Никишкин В.А. $C^{\infty}$-эквивалентность систем дифференциальных уравнений в окрестности особой точки типа "вырожденный узел" // Вестн. МГУ. Сер. 1. Матем., мех. 1976. №1. C. $27-35$.

[4] Бибиков Ю. Н. О приводимости системы двух дифференциальных уравнений к нормальной форме // Дифференц. уравнения. 1971. Т. 7. № 10. С. 1899-1902.

[5] Бибиков Ю.Н. Об одном критическом случае в теории устойчивости движения // Дифференц. уравнения. 1973. Т. 9. №12. С. 2123-2135.

[6] Кузнецов А.Н. Дифференцируемые решения вырождающихся систем обыкновенных уравнений // Функцион. анализ и его прилож. 1972. Т. 6. № 2. С. 41-51.

[7] Самовол В. С. О необходимом и достаточном условии гладкой линеаризации автономной системы на плоскости в окрестности особой точки // Матем. заметки. 1989. Т. 46. № 1. C. $67-77$.

Государственный университет "Высшая школа экономики", г. Москва

Поступило

E-mail: samovol@sshop.ru

12.11 .2003 